\title{
LA VEGETACIÓN DEL PEÑÓN DE GIBRALTAR
}

\author{
Antonio GALÁN DE MERA, John E. CORTÉS e Íñigo SÁNCHEZ GARCÍA
}

\begin{abstract}
RESUMEN. La vegetación del Peñón de Gibraltar. En el presente trabajo, presentamos un estudio de la vegetación de Gibraltar según la metodología de Braun-Blanquet. Como resultado, proponemos los nuevos sintáxones que aparecen en el resumen en inglés. La principal característica del paisaje vegetal de Gibraltar es que la vegetación natural está muy alterada con la presencia de comunidades fragmentarias. Éstas son comentadas aplicando los conceptos de 'comunidad basal' (BC), 'comunidad derivada' (DC) y 'comunidad marginal' (MC).
\end{abstract}

Palabras clave. Vegetación, Fitosociología, Gibraltar, Península Ibérica.

SUMMARY. The vegetation of the Rock of Gibraltar. In this work, we present a study of the vegetation of Gibraltar, using the Braun-Blanquet methodology. As a result, we propose the following new syntaxa: Anacyclo radiati-Hordeetum leporini centaureetosum sonchifoliae, Antirrhino cirrhigeri-Parietarietum judaicae and cymbalarietosum muralis, Asparago aphylli-Calicotometum villosae efedretosum fragilis, Ferulo tingitanae-Carthametum arborescentis and smyrnietosum olusatri, Geranio pusilli-Theligonetum cynocrambis geranietosum purpurei, Limonietum emarginati limonietosum sinuati, Oryzopsio miliaceiDaucetum maximi glaucietosum flavi, Parapholido incurvae-Frankenietum laevis, and Verbasco martineziiOnonidetum ramosissimae feruletosum tingitanae. The vegetation of Gibraltar is very perturbed, and the principal characteristic of the landscape are the fragmentary communities. These are commented, taking the 'basal community' (BC), 'derived community' (DC) and 'marginal community' (MC) concepts.

Key words. Vegetation, Phytosociology, Gibraltar, Iberian Peninsula.

\section{INTRODUCCIÓN}

El Peñón de Gibraltar (Gran Bretaña) (5,8 $\mathrm{Km}^{2}, 426 \mathrm{~m}$ ) está situado en el suroeste de la Península Ibérica $\left(36^{\circ} 40^{\prime} \mathrm{N}-5^{\circ} 40^{\prime} \mathrm{O}\right)$ en el extremo meridional de la provincia de Cádiz (España), cerrando hacia el este a la Bahía de Algeciras. Geológicamente es un promontorio perteneciente al arco calizo jurásico bético- rifeño (Didon et al., 1973) que quedó aislado por la formación mioceno-oligocena de las areniscas del Aljibe y por la apertura del Estrecho de Gibraltar a principios del Plioceno (Kiefer \& Bocquet, 1979). En la actualidad, es un tómbolo al haberse formado un istmo arenoso cuaternario que une el Peñón con la Península Ibérica (Meléndez \& Fuster, 1984). Además, su estructura geológica aparece 
dibujada por fallas que le dan un aspecto escarpado característico y depósitos arenosos de origen eólico al este y al oeste («Alameda and Catalan sands»)(Rose \& Rosenbaum, 1990).

Utilizando la tipología bioclimática de Rivas-Martínez et al. (1991), Gibraltar disfruta de bioclima termomediterráneo inferior (It = 426) subhúmedo (768 mm) de carácter oceánico $(\mathrm{Ic}=30)$. Podemos considerar bioindicadores termomediterráneos existentes en su flora: Acanthus mollis subsp. platyphyllos, Aristolochia baetica, Asparagus albus, Calicotome villosa, Ceratonia siliqua, Chamaerops humilis, Osyris quadripartita y Silene tomentosa (la nomenclatura y autoría de los táxones está basada en Castroviejo, 19861999; Valdés et al., 1987, y Linares et al., 1996).

El interés del estudio de la vegetación de este territorio está en la proximidad fitogeográfica que presenta con el norte de África, el Parque Natural Los Alcornocales (Pérez Latorre et al., 1999), y con otras áreas calizas del interior de la provincia de Cádiz (Sierra de las Cabras, Sierra de la Sal, Peña Arpada, Peña del Almed) y de Málaga (Sierra Crestellina). Entre ambas «Columnas de Hércules» (Peñón de Gibraltar y Jbel Moussa) podemos encontrar elementos florísticos comunes (Debeaux \& Dautez, 1889; Jahandiez \& Maire, 1931-1941; Deil, 1994; Linares et al., 1996)-Coronilla valentina subsp. glauca, Ephedra fragilis, Euphorbia squamigera, Ferula tingitana, Iberis gibraltarica, Limonium emarginatum, Prasium majus, Succowia balearica y Thymus willdenowii- que están indicando que el Peñón de Gibraltar es un pequeño territorio del Rif dentro de la Península Ibérica. Sobre todo dos grupos de táxones ponen aún más de manifiesto la relación que existe entre las áreas calcáreas de ambos lados del Estrecho de Gibraltar: Hypochaeris aggr. alliatae y Silene sect. Siphonomorpha. Hypochaeris alliatae (Biv.-Bern.) Galán de
Mera, Castro \& Vicente Orellana es una especie sobre todo norteafricana (Galán de Mera et al., 1999 a) que llega hasta el Jbel Moussa y hasta las sierras béticas de Córdoba utilizando probablemente el Peñón de Gibraltar (aunque aún aquí no se ha encontrado). Silene andryalifolia está repartida por todo el arco bético-rifeño (Jeanmonod, 1984), pero precisamente en el área del Estrecho de Gibraltar es uno de los lugares donde aparece una mayor variabilidad genética dentro de la sección Siphonomorpha: S. tomentosa (único endemismo de Gibraltar; Cortés \& Linares, 1993), S. gazulensis (del interior de la provincia de Cádiz; Galán de Mera et al., 1999 b), y $S$. rosulata x $S$. andryalifolia (en los alrededores de Tanger; Deil, 1994).

Otras plantas como Carthamus arborescens, Euphorbia squamigera, Ferula tingitana, Helichrysum rupestre, Prasium majus y Succowia balearica relacionan la flora del Peñón de Gibraltar con la de los territorios calizos más orientales de la Península Ibérica (Valdés et al., 1987) y del norte de África (Jahandiez \& Maire, 1931-1941).

Por su posición geográfica, la caracterización fitogeográfica del Peñón de Gibraltar quedaría de la siguiente forma (Pérez Latorre et al., 1996):

Región Mediterránea

Subregión Mediterránea Occidental

Superprovincia Iberomarroquí-Atlántica Provincia Tingitano-Onubo-Algarviense Sector Aljíbico

Subsector Algecireño

Distrito Gibraltareño

\section{MATERIAL Y MÉTODOS}

Gibraltar es un isleo calcáreo cuya vegetación se ha visto fuertemente influida por la presencia humana desde el s. VIII (Cortés, 1996) sobre todo con prácticas de pastoreo. 
Pero el uso del territorio es diferente al del resto de la Península Ibérica desde su ocupación inglesa en 1704, en que pasó a ser una plaza militar aislada del entorno, con lo que su vegetación leñosa se utilizó como fuente de energía (Kelaart, 1846). A principios del s. XIX comienzan a cultivarse plantas exóticas, con objeto de restaurar la masa forestal del territorio, que hoy día entran a formar buena parte de las comunidades vegetales naturales. El crecimiento humano en la ciudad de Gibraltar desde la Segunda Guerra Mundial, con un carácter marcadamente cosmopolita, ha aumentado la degradación de su vegetación a favor de la expansión de una flora alóctona de muy diferentes orígenes (Cortés \& Finlayson, 1988).

La vegetación del Peñón de Gibraltar es, pues, un ejemplo claro de isla invadida agresivamente por especies exóticas que dan lugar a comunidades fragmentarias, que hasta ahora, a pesar de los trabajos de otros autores (Kitayama \& Mueller-Dombois, 1995), han sido escasamente estudiadas en la Cuenca Mediterránea. El estado de la vegetación de Gibraltar exige, por tanto, la integración de los conceptos de «comunidad basal» (BC), «comunidad derivada» (DC) y «comunidad marginal» (MC) (Foucault, 1981; Dierschke, 1993; Kopecký et al., 1995) en el método fitosociológico (Braun-Blanquet, 1964). Una «comunidad basal» es la vegetación que comienza a originarse por invasión de un biótopo creado por la acción del hombre, siempre y cuando no se hayan alterado las características físico-químicas del suelo; y su composición florística recoge las plantas de las unidades sintaxonómicas de mayor rango. La «comunidad derivada» significa la invasión de una asociación por plantas diferentes a sus características, que a su vez, comienzan a disminuir su presencia. Para que esto suceda se han debido modificar profundamente las características físico-químicas del suelo; por ejemplo, con la instalación de cultivos. Cuando una comunidad derivada se ha establecido, las plantas características de la clase fitosociológica natural, forman grupos en biótopos favorables, constituyendo una comunidad marginal.

Dentro de la variabilidad de las asociaciones seguimos a Matuszkiewicz \& Matuszkiewicz (1981), que consideran a la vegetación como un contínuo relativo y concluyen con que la asociación fitosociológica recoge 3 variantes: 1- Variabilidad horizontalreferida a las razas geográficas, 2- Variabilidad vertical-referida a las formas altitudinales, y 3- Variabilidad local- que son las diferencias ecológicas (edáficas), y para ellas reservan el concepto de subasociación.

El presente trabajo está basado en 91 inventarios fitosociológicos (Braun-Blanquet, 1964); algunos, representativos de cada tipo de vegetación, se indican en la figura 1.

\section{RESULTADOS}

\section{Acebuchales}

Tamo communis-Oleetum sylvestris Benabid ex Pérez Latorre, Galán de Mera, Deil \& Cabezudo 1996; oleetosum sylvestris, fraxinetosum angustifoliae Benabid ex Pérez Latorre, Galán de Mera, Deil \& Cabezudo 1996 [Tabla 1]

Al igual que en otras áreas arcillosas del SO de la Península Ibérica (Pérez Latorre et al., 1996), los acebuchales constituyen en el Peñón de Gibraltar los bosques de enclaves sobre suelos profundos de altitudes medias (100-200 m). Su composición florística, sin embargo, es más próxima a los del norte de África (Benabid, 1984) que a los de la cercana Campiña Gaditana, donde se encuentran más empobrecidos (Galán de Mera, 1993). De esta forma, son especies diferenciales de Gibraltar y comunes con el norte de África: Ephedra fragilis, Fraxinus angustifolia, Genista 


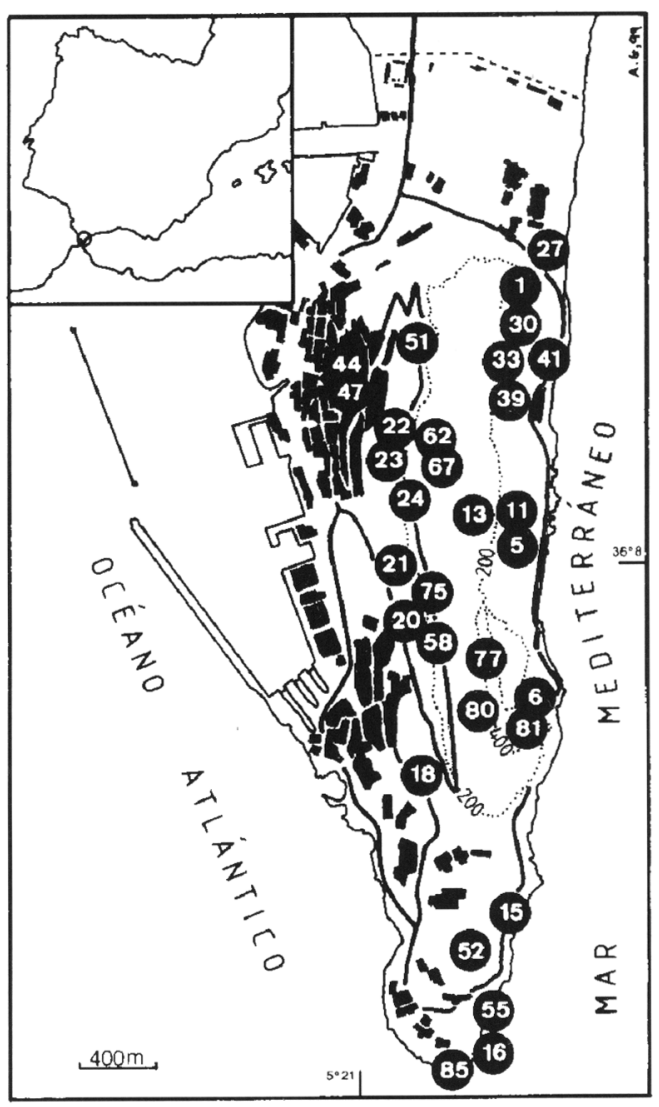

Figura 1. Mapa de Gibraltar con algunos inventarios representados en las tablas fitosociológicas. Map of Gibraltar with some releves represented in the phytosociological tables: Aloe arborescens DC- 52; Anacyclo radiati-Hordeetum leporini centaureetosum sonchifoliae-39, chrysanthemetosum coronarii- 51; Antirrhino cirrhigeri-Parietarietum judaicae- 44 y 47 , cymbalarietosum muralis- 20 y 21; Asparago albiRhamnetum oleoidis- 6, 80; Asparago aphylliCalicotometum villosae efedretosum fragilis- 30 y 33 , genistetosum linifoliae-24, 75; Biscutello sempervirentisIberidetum gibraltaricae- 13, 77 y 81; CistoMicromerietea MC- 62; Ferulo-Carthametum arborescentis smyrnietosum olusatri-58; Geranio pusilliTheligonetum cynocrambis geranietosum purpurei, raza geográfica con Succowia balearica-11; Limonietum emarginati-16, limonietosum sinuati- 85 , helichrysetosum rupestris- 41; Parapholido incurvae-Frankenietum laevis15 y 55; Polygono-Poetea annuae BC- 27; Tamo-Oleetum fraxinetosum angustifoliae-18, 22 y 23; TheroBrachypodietea MC- 67; Verbasco-Ononidetum ramosissimae feruletosum tingitanae- 1 y 5. linifolia, Laurus nobilis, Prasium majus y Ruscus hypophyllum. En suelos más arenosos y húmedos podemos observar la subasociación fraxinetosum angustifoliae, cuyas diferenciales son Fraxinus angustifolia y Ruscus hypophyllum (fig. 2). Sin embargo, los acebuchales de Gibraltar, presentan la originalidad de llevar en su composición florística Pistacia terebinthus, planta asociada a los suelos superficiales pedregosos y que define una variante del distrito Gibraltareño ya descrita anteriormente (Deil, 1994; Pérez Latorre et al., 1996).

La degradación de estos acebuchales, por introducción de ganadería o por construcciones urbanas, conduce a los lentiscares de PistacioRhamnetalia aunque, cuando la alteración es profunda, bajo los árboles encontramos plantas indicadoras de medios urbanos nitrificados, como Achyranthes sicula y Ferula tingitana.

\section{Lentiscares y espinales}

Asparago albi-Rhamnetum oleoidis Rivas Goday 1959

Asparago aphylli-Calicotometum villosae Rivas Goday ex Rivas-Martínez 1975; calicotometosum villosae, ephedretosum fragilis Galán de Mera, Cortés \& Sánchez García subass. nova

[Tabla 2; sintypus subass.: inv. 33]

En Gibraltar, la asociación Asparago albiRhamnetum oleoidis es poco abundante debido a la escasez de sustratos compactos. Sin embargo, es posible distinguir algunas de sus características (Asparagus albus, Jasminum fruticans, Rhamnus oleoides) frente al Asparago aphylli-Calicotometum villosae (Rivas-Martínez, 1974; Rivas-Martínez et al., 1990; Pérez Latorre et al., 1993).

Asparago-Calicotometum es un espinal cerrado dominado por Calicotome villosa y lentiscos que se asienta sobre suelos arenosos muy empobrecidos en nutrientes. Aunque la distribución de esta asociación es sólo 
tingitano-onubo-algarviense (Barbéro et al., 1981; Rivas-Martínez et al., 1990) existen ya bastantes datos sobre su variabilidad. En la provincia de Cádiz, se presenta la subasociación típica, la subasociación genistetosum linifoliae sobre areniscas (Pérez Latorre et al., 1993), y en el litoral la subasociación juniperetosum turbinatae (Pérez Latorre et al., 1996); de la Península Tingitana fue descrita la subasociación ampelodesmetosum mauritanicae (Galán de Mera, 1995). En el peñón de Gibraltar volvemos a encontrar a la subasociación ombrófila con genisteas (genistetosum linifoliae) y dos nuevos aspectos: ephedretosum fragilis subass. nova de localidades más xéricas y expuestas a los vientos de Levante (fig. 2), y una variante con Pistacia terebinthus, propia de áreas con suelos pedregosos.

Con excepción de AsparagoCalicotometum ephedretosum fragilis, que se manifiesta como vegetación permanente, en Gibraltar la asociación se comporta como una etapa de sustitución de los acebuchales que se asientan sobre suelos superficialmente arenosos. Este aspecto sucesional del TamoOleetum también destaca en ciertas áreas arenosas del interior de la provincia de Cádiz (Vejer de la Frontera) y de la Península Tingitana (Benabid, 1984).

La estructura de los acebuchales y de las comunidades con Calicotome villosa se altera por la introducción de cultivos como Acacia cyclops y Phoenix canariensis, aumentando la entrada en estas comunidades de plantas de carácter antropógeno, como Achyranthes sicula, Ferula tingitana o Urtica membranacea. El cultivo modifica las propiedades del suelo y las características de la fitocenosis, lo que interpretamos como comunidades derivadas.

Matorrales y espartales

Cisto-Micromerietea MC y TheroBrachypodietea MC
[Tabla 2]

Los matorrales y espartales sobre sustratos calizos se encuentran muy localizados en el territorio estudiado, sobre todo en áreas donde las comunidades nitrófilas aún no han desplazado del todo a la vegetación natural.

Los matorrales de la clase basófila CistoMicromerietea se encuentran muy empobrecidos, aunque plantas como Lavandula dentata, Lotus collinus, Ruta angustifolia y Teucrium lusitanicum relacionan a estas comunidades con los tomillares basófilos del S de Portugal (Rivas-Martínez et al., 1990), N de África (Deil \& Hammoumi, 1997), y áreas béticas más térmicas (Rivas Goday \& RivasMartínez, 1967). Lotus collinus (= L. creticus subsp. longisiliquosus R. Roemer) está ampliamente repartido por la Cuenca Mediterránea (Greuter et al., 1989) siendo el Peñón de Gibraltar su localidad europea más occidental (Valdés et al., 1987), lo que hace que estas comunidades de Cisto-Micromerietea estén sobre todo relacionadas con las del norte de África (Deil, 1994).

Los espartales se desarrollan sobre suelos

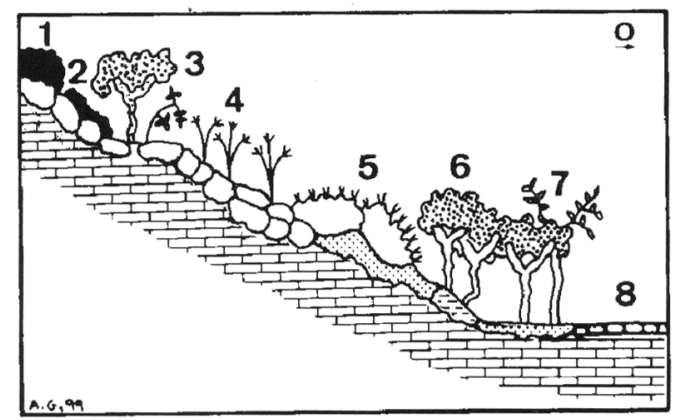

Figura 2. Esquema de la vegetación en la vertiente oeste del Peñón de Gibraltar. Vegetation scheme in the western side of the Rock of Gibraltar: 1Asparago albi-Rhamnetum oleoidis; 2- CistoMicromerietea MC; 3-Tamo-Oleetum sylvestris con Pistacia terebinthus; 4- Ferulo tingitanaeCarthametum arborescentis; 5- Asparago aphylliCalicotometum villosae ephedretosum fragilis; 6Tamo-Oleetum sylvestris; 7- Tamo-Oleetum sylvestris fraxinetosum angustifoliae; 8- Carretera. 
profundos, aunque como características de la clase Thero-Brachypodietea sólo podemos destacar a Euphorbia squamigera y Stipa tenacissima, indicando que forman una comunidad marginal. Euphorbia squamigera está presente en el S y SE de la Península Ibérica y NO de Africa (Castroviejo et al., 1986-1999), lo que nos lleva a pensar que las comunidades de Gibraltar están relacionadas con las ya descritas en los medios pedregosos del SE de España (Euphorbio squamigeraePhagnaletum almeriensis Rivas Goday \& Esteve 1972 y Euphorbio squamigeraeDianthetum valentini De la Torre \& Alcaraz in Alonso Vargas et al. 1998), pero no podemos adscribirlas a ninguna asociación ante la ausencia de plantas características (Alonso Vargas et al., 1998).

Las comunidades nitrófilas ligadas al medio urbano

Urtico dubiae-Smyrnietum olusatri (A. \& O. Bolòs 1950) O. Bolòs \& Molinier 1958, raza geográfica con Achyranthes sicula

[Tabla 3]

Esta comunidad es relativamente frecuente en el S de la Península Ibérica (Galán de Mera, 1993) y es la más urbanícola de las asociaciones de megaforbios de la Cuenca Mediterránea Occidental (Bolòs, 1962), ocupando sobre todo los suelos frescos y ahuecados de ambientes abandonados, umbríos y próximos a viviendas, como solares, escombreras y aceras. En Gibraltar y algunos puntos del S de la provincia de Cádiz (Castroviejo et al., 1986-1999) esta asociación se enriquece en el neófito tropical Achyranthes sicula que tiene una especial preferencia por estos medios y nos permite describir esta raza geográfica, que igualmente se puede localizar en otros puntos de las provincias Bética y Tingitano-OnuboAlgarviense (Bartolomé et al., 1989).

Geranio pusilli-Theligonetum cynocrambis geranietosum purpurei (Peinado, MartínezParras \& Bartolomé 1986) comb. \& stat. nov., raza geográfica con Succowia balearica

[Tabla 3]

La alianza Geranio-Anthriscion muestra una cierta complejidad en las sierras béticas y en el $\mathrm{N}$ de África, pues hasta el momento se han descrito numerosas comunidades cuya composición florística es bastante similar, e incluso presentan dificultades al pensar en su encuadre sintaxonómico: Geranio pusilliTheligonetum cynocrambis Rivas-Martínez \& Malato Beliz in Rivas-Martínez 1978 (iberomarroquí-atlántica, termomediterránea), Fumario sepii-Geranietum purpurei Peinado, Martínez-Parras \& Bartolomé 1986 (tingitanoonubo-algarviense, termomediterránea), Geranio purpurei-Galietum minutuli RivasMartínez \& Costa in Rivas-Martínez et al. 1980 (gaditano-onubense, termomediterránea), comunidades de Geranium lucidum y $G$. purpureum Pérez Latorre et al. 1998 (rondeñas), Succowio-Ceratocapnetum heterocarpae Daumas, Quézel \& Santa 1952 (oranense), Parietario mauritanicaeCeratocapnetum heterocarpae Martínez Parras 1982 (bética y murciano-almeriense, termomediterránea), Urtico dubiaeAnthriscetum caucalidis Rivas-Martínez et al. 1980 (onubense, termomediterránea) y Mercurialidi ellipticae-Theligonetum cynocrambis (Fernández Casas 1972) Peinado, Martínez-Parras \& Bartolomé 1986 (bética, termomediterránea).

En el norte de África y sur de la Península Ibérica, Deil (1994) y Deil \& Hammoumi (1997) aluden a una comunidad de Mercurialis ambigua y Theligonum cynocrambe que habita las fisuras de los roquedos y de los muros nitrificados. Este tipo de vegetación es un aspecto diferente, de clara tendencia fisurícola, del Geranio-Anthriscion. A juzgar por la composición florística de los inventarios que hemos levantado en la provincia de Cádiz y la Península Tingitana (Galán de Mera, 1993), 
las comunidades escionitrófilas con Theligonum cynocrambe parecen un aspecto basófilo, más petrano, del Fumario sepiiGeranietum purpurei lo que nos lleva a proponer el sintáxon Geranio pusilliTheligonetum cynocrambis geranietosum purpurei (de acuerdo con el artículo $25 \mathrm{del}$ CINF, mantenemos el nombre Geranio pusilliTheligonetum cynocrambis frente a Fumario sepii-Geranietum purpurei porque el primero es prioritario al ser el más antiguo y tratarse de reunión de sintáxones). Su significado ecológico subrupícola además se apoya en otras comunidades del Geranio-Anthriscion que también llevan casmófitos, como Stachys circinata (Fernández Casas, 1972).

Por otra parte, Succowia balearica es frecuente en los pastizales ombrófilos ligados a las calizas de la Cuenca Mediterránea próximas al mar: Succowio-Ceratocapnetum heterocarpae (Orán; Daumas et al., 1952), Succowio-Smyrnietum (Sicilia; Deil, 1994), Parietarietum judaicae sonchetosum dianae (SE de la Península Ibérica; Cantó et al., 1986),

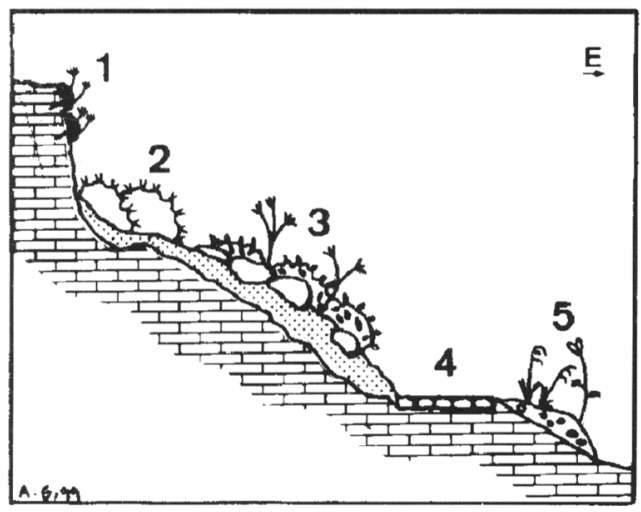

Figura 3. Esquema de la vegetación en la vertiente este del Peñón de Gibraltar. Vegetation scheme in the eastern side of the Rock of Gibraltar: 1Biscutello sempervirentis-Iberidetum gibraltaricae; 2- Asparago aphylli-Calicotometum villosae; 3Verbasco martinezii-Ononidetum ramosissimae feruletosum tingitanae; 4- Carretera; 5-OryzopsioDaucetum maximi glaucietosum flavi.
Comunidades con Succowia balearica (Bokkoya; Deil \& Hammoumi, 1997) y Parietario mauritanicae-Ceratocapnetum heterocarpae succowietosum balearicae (Granada, Almería; Martínez Parras, 1982). Se trata de una crucífera de dispersión exozoócora asociada probablemente a la ganadería caprina, que marca razas geográficas de las distintas asociaciones en el occidente de la Cuenca Mediterránea. En la provincia de Cádiz y en la Península Tingitana la asociación más extendida del Geranio-Anthriscion es Fumario sepii-Geranietum purpurei con una raza geográfica de Succowia balearica en la base de los roquedos calizos del litoral.

\section{Oryzopsio-Daucetum maximi glaucietosum} flavi Galán de Mera, Cortés \& Sánchez García subass. nova

[Tabla 3, sintypus subass.: inv. 38]

La asociación Oryzopsio-Daucetum maximi es frecuente en áreas termomediterráneas del Mediterráneo Occidental con influencia marítima. Normalmente se asienta en suelos que son arenosos en superficie, como bordes de carreteras o escombreras donde el sustrato está compactado. Si el suelo es arenoso-guijarroso, procedente de playas, se instala Glaucium flavum (Cantó et al., 1986) lo que nos permite describir esta nueva subasociación (fig. 3).

Ferulo tingitanae-Carthametum arborescentis Galán de Mera, Cortés \& Sánchez García ass. nova.; carthametosum arborescentis, smyrnietosum olusatri Galán de Mera, Cortés \& Sánchez García subass. nova

[Tabla 3 ; sintypus ass.: inv. 25, sintypus subass.: inv. 59]

Varias comunidades nitrófilas con Acanthus mollis subsp. platyphyllos han sido ya descritas en la Cuenca Mediterránea: Acantho-Smyrnietum olusatri (Sicilia; Brullo \& Marcenò, 1985), Urtico caudatae- 
Smyrnietum acanthetosum (Cerdeña; Biondi, 1989), Acantho-Smyrnietum olusatri carthametosum arborescentis (Gibraltar; Deil, 1994). Sin embargo, en Gibraltar Carthamus arborescens ofrece un carácter particular a este tipo de comunidades, tratándose de la localidad más occidental mediterránea de esta planta (Valdés et al., 1987). Además, AcanthoSmyrnietum olusatri carthametosum arborescentis está basada en una asociación siciliana, por lo que proponemos Ferulo tingitanae-Carthametum arborescentis en el distrito Gibraltareño (fig. 2). Ésta representa vegetación heliófila, nitrófila, de suelos calcáreos constantemente removidos, comportándose como etapa de sustitución antrópica de los espartales de Stipa tenacissima (Thero-Brachypodietea MC). Al tratarse de una comunidad de lugares soleados no se puede incluir en la alianza Allion triquetri O. Bolòs 1957, como sugiere Deil (1994). Asimismo, su posición ecológica es diferente a la asociación escionitrófila Acantho-Smyrnietum olusatri, que lleva en su composición florística plantas propias de medios sombreados (Allium triquetrum, Arisarum simorrhinum, Galium

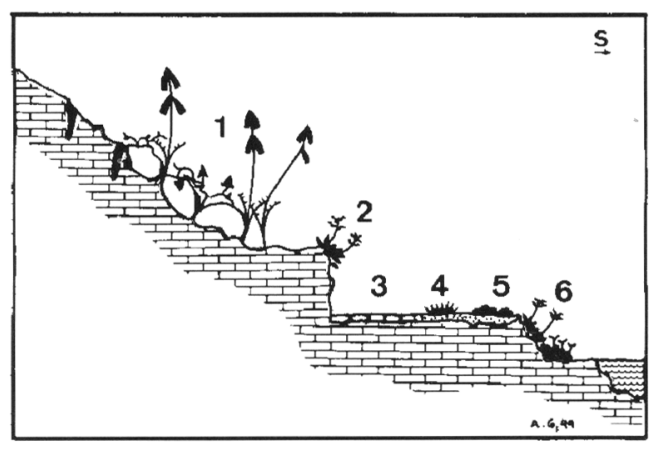

Figura 4. Esquema de la vegetación en la vertiente sur del Peñón de Gibraltar. Vegetation scheme in the southern side of the Rock of Gibraltar: 1-Aloe arborescens DC; 2- Limonietum emarginati; 3Carretera; 4- Polygono-Poetea annuae BC; 5Parapholido incurvae-Frankenietum laevis; 6Limonietum emarginati limonietosum sinuati. aparine, Parietaria judaica, Scrophularia peregrina y Urtica membranacea). Por tanto, la nueva asociación debe quedar dentro de la alianza Bromo-Oryzopsion (Foeniculum vulgare subsp. piperitum, Piptatherum miliaceum, Psoralea bituminosa) y del orden Ononidetalia ramosissimae. Los inventarios 25, 65, 68 y 72 pertenecen a áreas más nitrificadas por la influencia humana y menos soleadas, y con ellos describimos la subasociación smyrnietosum olusatri subass. nova.

Ferulo-Carthametum parece bastante próxima a Balloto hirsutae-Lavateretum maritimae (Cantó et al., 1986) y a Balloto hirsutae-Carthametum arborescentis (Rivas Goday \& Rigual, 1958), ambas del SE de la Península Ibérica. Estos autores incluyen a estas asociaciones en la clase de matorrales nitrohalófilos Pegano-Salsoletea Br.-B1. \& O. Bolòs 1958, aunque a juzgar por su composición florística deberían incluirse en el orden Ononidetalia ramosissimae de la clase Artemisietea vulgaris.

Verbasco martinezii-Ononidetum ramosissimae feruletosum tingitanae Galán de Mera, Cortés \& Sánchez García subass. nova

[Tabla 4, sintypus subass.: inv. 29]

Las características propias del orden Ononidetalia ramosissimae se hacen todavía más patentes con la asociación nitro-psamófila Verbasco-Ononidetum ramosissimae de la costa gaditana (Galán de Mera et al., 1997) y que ahora extendemos hasta el distrito Gibraltareño. En esta localidad aparece enriquecida con Ferula tingitana debido a los derrubios calizos que le confieren un carácter más alcalino al suelo, con lo que aportamos una nueva subasociación para el sur de la Península Ibérica (fig. 3). En el Peñón de Gibraltar estas comunidades vivaces destacan en los sedimentos eólicos de gran inclinación debidos a los vientos de Levante. 
Las comunidades viarias

Anacyclo radiati-Hordeetum leporini chrysanthemetosum coronarii Rivas-Martínez 1978, centaureetosum sonchifoliae Galán de Mera, Cortés \& Sánchez García subass. nova

[Tabla 5, sintypus subass.: inv. 39]

Asociación viaria, nitrófila y termófila, caracterizada por el endemismo occidental ibérico y tingitano Anacyclus radiatus, tratándose de una comunidad termomesomediterránea tingitano-onuboalgarviense, bética y luso-extremadurense. En el Peñón de Gibraltar los suelos de naturaleza caliza permiten la instalación de las plantas propias de chrysanthemetosum coronarii (Rivas-Martínez, 1978); en cambio, los medios enriquecidos con arenas marítimas inducen al desarrollo de plantas como Centaurea sonchifolia, Emex spinosa o Lotus creticus, que interpretamos como la nueva subasociación centaureetosum sonchifoliae. Su significado ecológico es además una transición entre las comunidades viarias de Hordeion leporini y la asociación nitro-psamófila mediterránea LotoCentaureetum sphaerocephalae Nègre 1964 (Géhu \& Sadki, 1995).

Anacyclo-Hordeetum chrysanthemetosum coronarii es sustituida en las provincias Catalano-Valenciano-Provenzal y Baleárica por la asociación Resedo-Chrysanthemetum (Bolòs \& Molinier, 1958), en la provincia Orano-Kabiliense por el ChrysanthemoConvolvuletum althaeoides (Nègre, 1964), y en la provincia Sícula por ei Malvo parvifloraeChrysanthemetum coronarii (Ferro, 1980).

En Gibraltar Anacyclo-Hordeetum leporini se encuentra bastante fragmentada e invadida por características de Chenopodietalia y Onopordenea. Debido a que el suelo está muy removido y nitrificado, aparece una tendencia a la disminución de las plantas de Hordeion leporini, por ello pensamos que los inventarios 34, 35, 36, 37 y 51 representan una comunidad derivada donde se hacen muy importantes plantas como Malva sylvestris, Oxalis pes-caprae, Urospermum picroides o Urtica membranacea.

Las comunidades halonitrófilas

Parapholido incurvae-Frankenietum laevis Galán de Mera, Cortés \& Sánchez García ass. nova

[Tabla 6; sintypus ass.: inv. 57]

Hasta el momento, las comunidades halófilas de Parapholis incurva se incluían dentro de la clase fitosociológica Saginetea maritimae Westhoff, Van Leeuwen \& Adriani 1962 (=Frankenietea pulverulentae RivasMartínez in Rivas-Martínez \& Costa 1976), cuyo significado ecológico es el de vegetación halonitrófila anual sometida a inundaciones temporales de agua salada o bien, a una maresía salina continuada (Rivas-Martínez et al., 1990; Galán de Mera et al., 1997). Sin embargo, tanto en la provincia de Cádiz, como en el norte de África, aparecen comunidades vivaces con Frankenia laevis, Parapholis incurva, $P$. filiformis, P. pycnantha, Plantago crassifolia, e incluso Juncus maritimus (Galán de Mera, 1993; Deil, 1997), lo cual nos lleva a considerar esta nueva asociación dentro de la clase Juncetea maritimi (fig. 4).

La abundancia de Frankenia laevis en las áreas salinas del sur de la Península Ibérica y norte de África (Nègre, 1957), fuera de los esteros (Salicornietea fruticosae Br.-Bl. \& R.Tx. 1943), separa esta nueva asociación del Plantagini coronopi-Hordeetum marini $\mathrm{O}$. Bolòs \& Molinier ex O. Bolòs 1962 y del Parapholido incurvae-Frankenietum pulverulentae Rivas-Martínez ex Castroviejo \& Porta 1976 (Rivas Goday, 1964; RivasMartínez et al., 1980).

Los bordes de las carreteras de Gibraltar o aquellos medios que son frecuentemente pisoteados presentan una vegetación terofítica dominada sobre todo por Polycarpon tetraphyllum (inv. 27 y 28). Puesto que el resto 
de los elementos de la clase Polygono-Poetea annuae están ausentes, podemos interpretar este hecho como una transición entre las comunidades de Frankenia laevis y las menos exigentes en sales de Polygono-Poetea, que comienzan a desarrollarse mediante una comunidad basal (fig. 4).

\section{Las comunidades rupícolas}

Limonietum emarginati Asensi Marfil 1984; limonietosum emarginati, helichrysetosum rupestris Deil 1994 corr., limonietosum sinuati Galán de Mera, Cortés \& Sánchez García subass. nova

[Tabla 7, sintypus subass.: inv. 85]

Se trata de una asociación de exigencias aerohalinas muy estrictas de roquedos expuestos al mar, con una distribución aljíbica, tingitana y rifeña (Asensi Marfil, 1984; Deil, 1994; Deil \& Hammoumi, 1997).

Según Deil (1994) dentro de su variabilidad se distinguen tres subasociaciones: spergularietosum fimbriatae Asensi Marfil 1984, carpobrotetosum edulis Deil 1994 y helichrysetosum rupestris Deil 1994 corr. (corregido aquí). La subasociación spergularietosum, descrita de la isla de Tarifa, es un aspecto de roquedos marítimos con menor inclinación en tránsito a ParapholidoFrankenietum laevis; la vegetación de Limonium emarginatum y Carpobrotus edulis podría tratarse más bien de una comunidad derivada, puesto que ésta última es un neófito invasor de origen sudafricano (Castroviejo et al., 1986-1999); y helichrysetosum rupestris es una subasociación de suelos más profundos, donde es posible el desarrollo de plantas leñosas.

En Gibraltar aún es posible observar nuevas formas de esta asociación. En aquellos medios más ruderalizados y ricos en arenas se introduce Limonium sinuatum, con lo que describimos la subasociación limonietosum sinuati (fig. 4). La ruderalización puede llegar a ser tan alta que plantas escapadas de cultivo (Senecio cineraria) acompañadas de otras, como Ecballium elaterium o Beta maritima, pueden desplazar a la vegetación natural constituyendo una comunidad derivada (Senecio cineraria DC). Este aspecto del Limonietum emarginati también fue aportado por Deil (1994) en los alrededores del Faro del Camarinal (Cádiz) (Limonium sinuatumCalendula carbonellii-Gesellschaft), y por nosotros mismos en la Punta del Carnero (Bahía de Algeciras).

En general, en Gibraltar, las comunidades rupícolas y glerícolas están muy fragmentadas por la invasión de plantas alóctonas que forman comunidades derivadas (Aeonium arboreum DC, Carpobrotus edulis DC) que impiden que las fitocenosis se desarrollen con todas sus características.

\section{Biscutello sempervirentis-Iberidetum gibraltaricae Deil 1994}

[Tabla 7]

Asociación endémica del Peñón de Gibraltar constituida por elementos ibéricos meridionales y norteafricanos que pueblan las fisuras verticales más elevadas. La especie que domina a la asociación es Iberis gibraltarica, aunque ésta también está presente en otros roquedos de Andalucía Occidental y del Rif (Valdés et al., 1987). Quizás la especie que da más carácter a la asociación es Silene tomentosa, endemismo gibraltareño ausente de los inventarios levantados hasta el momento. Otras plantas, como Biscutella sempervirens, Cerastium gibraltaricum y Saxifraga globulifera están ampliamente repartidas por las Sierras Béticas (Castroviejo et al., 19861999), lo que permite la inclusión de esta asociación en la alianza Campanulion velutinae (Pérez Latorre et al., 1996) del suborden Tinguarrenalia siculae (fig. 3).

Phagnalo-Rumicetea indurati (Rivas Goday \& Esteve 1972) Rivas-Martínez, Izco \& Costa 


\section{3, Aloe arborescens DC}

[Tabla 7]

Los derrubios causados por la erosión natural y antrópica de la roca de Gibraltar, albergan a las plantas características de esta clase fitosociológica, sobre todo Rumex induratus y Echium creticum subsp. coincyanum. Aunque ésta última también aparece en otros roquedos calizos y dolomíticos andaluces (Galán de Mera, 1993), en nuestro territorio, las comunidades están tan empobrecidas, que no podemos adscribirlas a ninguna asociación. Agave americana, Aloe arborescens y Tanacetum cinerarifolium invaden las gleras y derrubios formando la comunidad derivada de Aloe arborescens (fig. 4).

\section{Asplenietea trichomanis $\mathrm{BC}$}

[Tabla 7]

En un pequeño talud umbrío y terroso orientado hacia el $\mathrm{E}$ hemos realizado un inventario con Asplenium trichomanes y Asplenium billotii. Las comunidades con Asplenium billotii son frecuentes en la Península Ibérica (Loidi \& Galán de Mera, 1988; García Río \& Navarro Andrés, 1994) acompañadas de plantas acidófilas, como Cheilanthes tinaei, Cystopteris fragilis y Sedum hirsutum. La pobreza de elementos encontrada en el Peñón nos induce a considerar, de nuevo, el concepto de comunidad basal.

\section{Anomodonto-Polypodietea BC}

\section{[Tabla 7]}

Aunque las comunidades mediterráneoatlánticas con Polypodium cambricum subsp. serrulatum son muy frecuentes en las áreas circundantes al Estrecho de Gibraltar (Galán de Mera et al., 1996), en el territorio estudiado sólo hemos podido levantar un inventario que correspondía al muro de una casa. En él, la ausencia de pteridófitos y líquenes que Bolòs (1957) incluye en el orden AnomodontoPolypodietalia O. Bolòs \& Vives in O. Bolòs
1957, nos lleva al concepto de comunidad basal como aspecto incipiente de la vegetación.

Antirrhino cirrhigeri-Parietarietum judaicae Galán de Mera, Cortés \& Sánchez García ass. nova; parietarietosum judaicae, cymbalarietosum muralis Galán de Mera, Cortés \& Sánchez García subass. nova

[Tabla 8; sintypus ass.: inv.47, sintypus subass.: inv. 48]

Vegetación de fisuras verticales de muros viejos, castillos y roquedos con una fuerte influencia antropozoógena.

Este tipo de vegetación es bastante diversa en las áreas calcáreas de la Península Ibérica, siendo más monótona en las cordilleras silíceas del interior (Rivas-Martínez, 1969; Ortiz \& Rodríguez-Oubiña, 1993). En el Peñón de Gibraltar y sus alrededores, las comunidades con Parietaria judaica están enriquecidas con plantas meridionales (Antirrhinum majus subsp. cirrhigerum, Fumaria capreolata, $F$. rupestris), lo que nos permite dar a conocer esta nueva asociación.

En las paredes que han sido alguna vez encaladas, rezumantes o con retención de aguas, se instala Trachelium caeruleum, y especialmente Cymbalaria muralis, manifestándose la transición hacia la clase Adiantetea Br.-Bl. in Br.-Bl., Roussine \& Nègre 1952, a través de la nueva subasociación cymbalarietosum muralis.

La nitrofilia que ofrecen estos medios facilita la naturalización de plantas exóticas que llegan a alcanzar un índice de abundancia elevado (Nephrolepis cordifolia DC, Tropaeolum majus DC).

\section{ESQUEMA SINTAXONÓMICO}

QUERCETEA ILICIS Br.-Bl. ex O. Bolòs 1950

+ Quercetalia ilicis Br.-B1. ex Molinier 1934 em. Rivas-Martínez 1975

* Querco rotundifoliae-Oleion sylvestris Barbéro, Quézel \& Rivas-Martínez in Rivas- 
Martínez, Costa \& Izco 1986

1. Tamo communis-Oleetum sylvestris Benabid ex Pérez Latorre, Galán de Mera, Deil \& Cabezudo 1996 [Acebuchales verticícolas].

oleetosum sylvestris

fraxinetosum angustifoliae Benabid ex Pérez Latorre, Galán de Mera, Deil \& Cabezudo 1996 [Acebuchales con fresnos]. variante con Pistacia terebinthus [Variante de suelos superficiales pedregosos].

+ Pistacio lentisci-Rhamnetalia alaterni RivasMartínez 1975

* Asparago albi-Rhamnion oleoidis Rivas Goday ex Rivas-Martínez 1975

2. Asparago albi-Rhamnetum oleoidis Rivas Goday 1959 [Lentiscares calcícolas de suelos compactos].

3. Asparago aphylli-Calicotometum villosae Rivas-Martínez 1975 [Espinales silicícolas].

calicotometosum villosae

genistetosum linifoliae Pérez Latorre, Nieto Caldera \& Cabezudo 1993 [Espinales ombrófilos con escobones].

ephedretosum fragilis Galán de Mera, Cortés \& Sánchez García subass. nova [Espinales xéricos].

variante con Pistacia terebinthus [Espinales de suelos pedregosos].

Acacia cyclops DC [Cultivos invasores de acacias].

Phoenix canariensis DC [Cultivos invasores de palmera canaria].

\section{CISTO-MICROMERIETEA JULIANAE} Oberdorfer 1954

4. Cisto-Micromerietea MC [Matorrales de sustratos calizos].

THERO-BRACHYPODIETEA Br.-Bl. ex A. \&

O. Bolòs 1950

5. Thero-Brachypodietea $\mathrm{MC}$ [Espartales de sustratos calizos].
GALIO APARINES-URTICETEA DIOICAE

Passarge ex Kopecký 1969

+ Glechometalia hederaceae R. Tx. in R. Tx. \& Brun-Hool 1975

* Alliarion petiolatae Oberdorfer (1957) 1962

6. Urtico dubiae-Smyrnietum olusatri (A. \& O. Bolòs 1950) O. Bolòs \& Molinier 1958 [Comunidades escionitrófilas de suelos húmedos].

Raza geográfica con Achyranthes sicula

STELLARIETEA MEDIAE R.Tx., Lohmeyer \& Preising ex von Rochow 1951

Stellarienea mediae

+ Sisymbrietalia officinalis J.Tx. in Lohmeyer et al. 1962 em. Rivas-Martínez, Báscones, Díaz, Fernández-González \& Loidi 1991

* Hordeion leporini Br.-B1. in Br.-B1., Gajewuski, Wraber \& Walas 1936

7. Anacyclo radiati-Hordeetum leporini $\mathrm{O}$. Bolòs \& Rivas-Martínez in Rivas-Martínez 1978 [Vegetación viaria nitrófila].

chrysanthemetosum coronarii RivasMartínez 1978 [Subasociación de suelos enriquecidos en bases].

centaureetosum sonchifoliae Galán de Mera, Cortés \& Sánchez García subass. nova [Subasociación de suelos enriquecidos en arenas marítimas].

Geranio purpurei-Cardaminenea hirsutae Rivas-Martínez, Fernández-González \& Loidi 1998

+ Geranio purpurei-Cardaminetalia hirsutae Brullo in Brullo \& Marcenò 1985

* Geranio pusilli-Anthriscion caucalidis RivasMartínez 1977

8. Geranio pusilli-Theligonetum cynocrambis Rivas-Martínez \& Malato Beliz in Rivas-Martínez 1978 [Comunidades terofíticas escionitrófilas y humícolas].

geranietosum purpurei (Peinado, Martínez-Parras \& Bartolomé 1986) Galán de Mera, Cortés \& Sánchez García comb. \& stat. nov. (Fumario sepii-Geranietum 
purpurei) [Subasociación basófila petrana]. Raza geográfica con Succowia balearica

ARTEMISIETEA VULGARIS Lohmeyer, Preising \& R.Tx. ex von Rochow 1951

Onopordenea acanthii Rivas-Martínez, Báscones, Díaz, Fernández-González \& Loidi 1991

+ Ononidetalia ramosissimae Galán de Mera, Sánchez García \& Vicente Orellana 1997

* Bromo-Oryzopsion miliacei O. Bolòs 1970

9. Oryzopsio miliacei-Daucetum maximi $\mathrm{O}$. Bolòs \& Vigo ex O. Bolòs 1975 [Altabacares termófilos].

glaucietosum flavi Galán de Mera, Cortés

\& Sánchez García subass. nova [Comunidad de suelos guijarrosos].

10. Ferulo tingitanae-Carthametum arborescentis Galán de Mera, Cortés \& Sánchez García ass. nova [Cardales heliófilos].

carthametosum arborescentis

smyrnietosum olusatri Galán de Mera, Cortés \& Sánchez García subass. nova [Subasociación de lugares menos soleados].

11.Verbasco martinezii-Ononidetum ramosissimae Galán de Mera, Sánchez García \& Vicente Orellana 1997 [Comunidad nitro-psammófila].

feruletosum tingitanae Galán de Mera, Cortés \& Sánchez García subass. nova [Aspecto de derrubios calcáreos].

JUNCETEA MARITIMI Br.-Bl. in Br.-Bl., Roussine \& Nègre 1952

+ Juncetalia maritimi Br.-Bl. ex Horvatic 1934

* Juncion maritimi Br.-Bl. ex Horvatic 1934

12. Parapholido incurvae-Frankenietum laevis Galán de Mera, Cortés \& Sánchez García ass. nova [Vegetación vivaz halonitrófila].

POLYGONO-POETEA ANNUAE RivasMartínez 1975

13. Polygono-Poetea annuae $\mathrm{BC}$ [Vegetación viaria de suelos pisoteados].

CRITHMO-STATICETEA Br.-B1. in Br.-Bl., Roussine \& Nègre 1952

+ Crithmo-Staticetalia Molinier 1934

* Crithmo-Daucion halophili Rivas-Martínez, Lousa, Díaz, Fernández-González \& Costa 1990

14. Limonietum emarginati Asensi Marfil 1984 [Comunidad aerohalófila de acantilados marítimos].

limonietosum emarginati

helichrysetosum rupestris Deil 1994 corr. (corregido aquí; helichrysetosum stoechadis)[Aspecto de roquedos marítimos poco inclinados].

limonietosum sinuati Galán de Mera, Cortés \& Sánchez García subass. nova [Subasociación de medios ruderalizados arenosos].

Aeonium arboreum DC [Cultivos invasores de siempreviva arbórea].

Carpobrotus edulis DC [Cultivos invasores de bálsamo].

Senecio cineraria DC [Cultivos invasores de cineraria].

ASPLENIETEA TRICHOMANIS (Br.-Bl. in Meier \& Br.-Bl. 1934) Oberdorfer 1977

+ Asplenietalia glandulosi Br.-Bl. in Meier \& Br.-Bl. 1934

++ Tinguarrenalia siculae (Daumas, Quézel \& Santa 1952) Galán de Mera in Pérez Latorre, Galán de Mera, Deil \& Cabezudo 1996

* Campanulion velutinae Martínez Parras \& Peinado Lorca 1990

15. Biscutello sempervirentis-Iberidetum gibraltaricae Deil 1994 [Comunidades fisurícolas de calizas].

16. Asplenietea trichomanis $\mathrm{BC}$ [Comunidades empobrecidas con helechos].

PHAGNALO-RUMICETEA INDURATI (Rivas Goday \& Esteve 1972) Rivas-Martínez, Izco \& Costa 1973 
Tabla 1

Tamo communis-Oleetum sylvestris oleetosum sylvestris, fraxinetosum angustifoliae (Quercetea ilicis, Quercetalia ilicis, Querco-Oleion sylvestris)

\begin{tabular}{llllllllll}
\hline Inventario $^{\circ}$ & 17 & 69 & 18 & 22 & 23 & 6 & 64 & 70 & 74 \\
Area m$^{2}$ & 100 & 100 & 100 & 100 & 100 & 100 & 100 & 100 & 100 \\
Orientación & $\mathrm{SE}$ & $\mathrm{O}$ & $\mathrm{O}$ & $\mathrm{O}$ & - & 0 & 0 & $\mathrm{O}$ & $\mathrm{O}$ \\
Pendiente $(\%)$ & 20 & 10 & 20 & 10 & 5 & 10 & 10 & 10 & 5
\end{tabular}

Características de asociación y alianza

Olea sylvestris

Tamus communis

Prasium majus

Arum italicum

Diferenciales de subasociación y variante

Ruscus hypophyllum

Acanthus platyphyllos

Laurus nobilis

Pistacia terebinthus

Fraxinus angustifolia

Hedera helix

Características de Pistacio-Rhamnetalia

Aristolochia baetica

Asparagus albus

Rhamnus alaternus

Osyris quadripartita

Asparagus aphyllus

Jasminum fruticans

Teucrium fruticans

Genista linifolia

Chamaerops humilis

Phlomis purpurea

Ephedra fragilis

Pistacia lentiscus

Calicotome villosa

Rhamnus oleoides

Características de Quercetalia ilicis y Quercetea ilicis

Clematis cirrhosa

Urginea maritima

Arisarum simorrhinum

Rubia longifolia

Smilax aspera

Lonicera implexa

Clematis flammula

Compañeras

Ferula tingitana

Asphodelus albus

Achyranthes sicula

Vinca difformis

Melica minuta

Geranium purpureum

Carthamus arborescens

Elaeoselinum foetidum

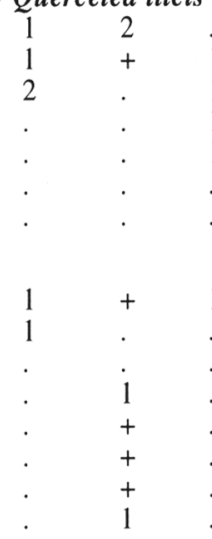

Otros táxones: Lotus sp. +, Pallenis spinosa +, Hyparrhenia podotricha 1 y Vicia lutea + en 17; Crataegus brevispina 2, Celtis australis 1, Lonicera hispanica + y Rubus ulmifolius 1 en 22; Brachypodium gaditanum 1 y Bryonia dioica 1 en 61; Annogramma leptophylla + en 69; Urtica membranacea 1, Smyrnium olusatrum + , Vitis sylvestris + Oxalis pes-caprae 2 y Parietaria judaica 1 en 74. 
Tabla 2

6-80: Asparago albi-Rhamnetum oleoidis ,32-75: Asparago aphylli-Calicotometum villosae calicotometosum villosae, genistetosum linifoliae, ephedretosum fragilis Galán de Mera, Cortés \& Sánchez García subass. nova (Quercetea ilicis, Pistacio-Rhamnetalia, Asparago-Rhamnion)

\begin{tabular}{lllllllllllllll}
\hline Inventario $\mathrm{n}^{\circ}$ & 6 & 73 & 80 & 32 & 10 & 7 & 8 & 30 & 33 & 24 & 75 & 62 & 67 & 76 \\
Área $\mathrm{m}^{2} 100$ & 50 & 100 & 100 & 100 & 40 & 50 & 50 & 50 & 60 & 100 & 5 & 20 & 100 & \\
Orientación & $\mathrm{O}$ & $\mathrm{O}$ & $\mathrm{O}$ & $\mathrm{NE}$ & $\mathrm{O}$ & $\mathrm{O}$ & $\mathrm{O}$ & $\mathrm{E}$ & $\mathrm{NE}$ & $\mathrm{O}$ & 0 & 0 & 0 & 0 \\
Pendiente (\%) & 40 & - & 55 & 40 & 30 & 40 & - & - & 30 & - & 60 & 40 & 70 & 50
\end{tabular}

Características de asociaciones y alianza

Calicotome villosa

Asparagus albus

Aristolochia baetica

Rhamnus oleoides

Asparagus aphyllus

Jasminum fruticans

Phlomis purpurea

$\begin{array}{llllllllllllll}+ & + & . & 4 & 3 & 1 & 1 & + & 4 & 3 & 1 & . & . & 1 \\ + & 1 & 1 & . & . & . & . & . & . & 1 & + & 1 & 1 & 1 \\ 1 & 1 & 2 & 1 & . & . & . & . & . & . & 1 & . & . & . \\ 1 & . & 1 & . & . & . & . & . & . & . & . & . & . & . \\ . & . & . & . & 1 & . & . & 1 & . & . & . & . & . & . \\ . & + & . & . & . & . & . & . & . & . & . & . & + & . \\ . & . & 1 & . & . & . & . & . & . & . & . & . & . & .\end{array}$

Diferenciales de subasociaciones y variante

Ephedra fragilis

Pistacia terebinthus

Genista linifolia

Características de Pistacio-Rhamnetalia

Pistacia lentiscus

Rhamnus alaternus

Osyris quadripartita

Coronilla glauca

Chamaerops humilis

Teucrium fruticans

Bupleurum fruticosum

Melica arrecta

Características de Quercetea ilicis

Rubia longifolia

Arisarum simorrhinum

Prasium majus

Lonicera implexa

Clematis cirrhosa

Olea sylvestris

Urginea maritima

Smilax aspera

Acacia cyclops DC

Acacia cyclops

Phoenix canariensis DC

Phoenix canariensis

Cisto-Micromerietea MC

Teucrium lusitanicum

Ruta angustifolia

Melica minuta

Lavandula dentata

Lotus collinus

Thero-Brachypodietea MC

Stipa tenacissima

Euphorbia squamigera

Compañeras

Asphodelus albus

Ferula tingitana

Tamus communis

Oxalis pes-caprae

Carthamus arborescens

Achyranthes sicula

Urtica membranacea

Centranthus calcitrapae

Vinca difformis

Lobularia maritima

$\begin{array}{llllllllllllll}4 & 3 & . & 3 & 2 & 4 & 3 & 4 & 1 & . & 4 & . & . & . \\ 2 & 1 & . & 1 & 1 & . & 2 & . & 1 & 4 & 2 & . & . & . \\ 1 & 1 & + & . & 2 & . & . & 2 & . & 1 & 1 & . & . & 1 \\ + & . & . & . & . & . & . & . & . & . & 1 & 1 & . & . \\ . & . & 3 & . & . & . & . & . & . & + & . & . & . & . \\ . & . & 1 & . & . & . & . & . & . & . & . & + & . & . \\ . & . & . & . & . & . & . & . & . & . & 2 & . & . & . \\ . & . & . & . & . & . & . & . & . & . & + & . & . & .\end{array}$

Otros táxones: Sonchus tenerrimus + en 7; Dactylis hispanica + y Daucus maximus + en 10; Succowia balearica + , Bromus diandrus 1 y Parietaria judaica 1 en 30; Geranium purpureum 1 y Geranium columbinum + en 32 ; Umbilicus rupestris +, Acanthus platyphyllos +, Kundmannia sicula + y Sedum album + en 75; Asteriscus maritimus 1, Hyparrhenia podotricha 1 y Avena barbata + en 76 ; Elaeoselinum foetidum 1, Geranium rotundifolium + y Magydaris panacifolia + en 80 
Tabla 3

9: Urtico dubiae-Smyrnietum olusatri, raza geográfica con Achyranthes sicula (Galio-Urticetea, Glechometalia, Alliarion petiolatae) 11: Geranio-Theligonetum cynocrambis geranietosum purpurei, raza geográfica con Succowia balearica (Stellarietea, GeranioCardaminenea, Geranio-Cardaminetalia, Geranio-Anthriscion)

38: Oryzopsio-Daucetum maximi glaucietosum flavi Galán de Mera, Cortés \& Sánchez García subass. nova (Artemisietea. Onopordenea. Ononidetalia ramosissimae, Bromo-Oryzopsion)

25-72: Ferulo tingitanae-Carthametum arborescentis Galán de Mera, Cortés \& Sánchez García ass. nova, carthametosum arborescentis, smyrnietosum olusatri Galán de Mera, Cortés \& Sánchez García subass. nova (Artemisietea, Onopordenea, Piptatheretalia, Bromo Oryzopsion)

\begin{tabular}{llllllllllllll}
\hline Inventario no $^{\circ}$ & 9 & 11 & 38 & 25 & 58 & 65 & 68 & 72 & 59 & 60 & 63 & 66 & 71 \\
Área m$^{2}$ & 5 & 2 & 50 & 50 & 10 & 50 & 20 & 50 & 50 & 10 & 20 & 20 & 20 \\
Orientación- & - & - & 0 & SO & 0 & 0 & 0 & 0 & 0 & 0 & 0 & 0 & 0 \\
Pendiente- & - & - & 40 & 10 & - & 50 & - & - & 55 & 75 & 50 & 70 & 80
\end{tabular}

Pendiente-

$40 \quad 10$

Urtico-Smyrnietum olusatri raza geográfica con Achyranthes sicula

Oxalis pes-caprae

Achyranthes sicula

Urtica membranacea

$\begin{array}{ll}2 & 1 \\ 3 & 2 \\ 2 & 1\end{array}$

Geranio-Theligonetum geranietosum purpurei raza geográfica

con Succowia balearica y Geranio-Anthriscion

Succowia balearica

Centranthus calcitrapae

Geranium purpureum

Fumaria sepium

Oryzopsio-Daucetum maximi glaucietosum flavi

Daucus maximus

Piptatherum miliaceum

Glaucium flavi

Dittrichia viscosa

Ferulo-Carthametum arborescentis

Acanthus platyphyllos

Ferula tingitana

Carthamus arborescens

smyrnietosum olusatri

Smyrnium olusatrum

Características de Bromo-Oryzopsion, Piptatheretalia y Onopordenea

Psoralea bituminosa

Hyparrhenia podotricha

Lavandula multifida

Nicotiana glauca

Scabiosa atropurpurea

Euphorbia terracina

Foeniculum piperitum

Características de Stellarietea mediae

Sonchus tenerrimus

Allium ampeloprasum

Lavatera arborea

Urospermum picroides

Sonchus oleraceus

Medicago polymorpha

Reseda alba

Erodium malacoides

Galactites tomentosa

Echium coincyanum

Malva sylvestris

Thero-Brachypodietea MC

Stipa tenacissima

Compañeras

Asparagus albus

Prasium majus

Olea sylvestris

Clematis cirrhosa

Osyris quadripartita

Asphodelus albus

Rhamnus alaternus

Teucrium fruticans

Melica minuta

Urginea maritima

Lavandula dentata

Coronilla glauca

Antirrhinum cirrhigerum

Rubia longifolia

Otros táxones: Arisarum simorrhinum + en 9; Parietaria diffusa 1 en 11; Calicotome villosa + en 25; Genista linifolia + en 38 Brachypodium gaditanum 1 y Crocosmia crocosmiflora + en 58; Ruta angustifolia + en 59; Sedum sediforme + en 60; Teucrium lusitanicum +, Lonicera implexa + y Phlomis purpurea + en 63; Asparagus aphyllus + en 66; Vinca difformis + y Tamus communis + en 68; Biscutella sempervirens + y Magydaris panacifolia + en 71; Hyoseris radiata + en 72. 
Tabla 4

Verbasco martinezii-Ononidetum ramosissimae feruletosum tingitanae

Galán de Mera, Cortés \& Sánchez García subass. nova

(Artemisietea, Onopordenea, Ononidetalia ramosissimae, Bromo-Oryzopsion)

\begin{tabular}{lllllll}
\hline Inventario $\mathrm{n}^{\circ}$ & 1 & 2 & 5 & 19 & 29 & 31 \\
Área $^{2}$ & 100 & 100 & 100 & 10 & 100 & 100 \\
Orientación & $\mathrm{E}$ & $\mathrm{E}$ & $\mathrm{E}$ & $\mathrm{O}$ & $\mathrm{E}$ & $\mathrm{E}$ \\
Pendiente $(\%)$ & 50 & 50 & 50 & - & 50 & 60
\end{tabular}

Características de asociación y unidades superiores

Ononis ramosissima

Psoralea bituminosa

Daucus maximus

Scabiosa atropurpurea

Foeniculum piperitum

Carpobrotus edulis

Lobularia maritima

Phagnalon saxatile

Carlina corymbosa

Verbascum martinezii

Convolvulus althaeoides

Dittrichia viscosa

Hyparrhenia podotricha

\begin{tabular}{|c|c|c|c|c|}
\hline 3 & 3 & 3 & & 2 \\
\hline 1 & 1 & 1 & 3 & . \\
\hline . & . & 1 & . & 1 \\
\hline . & + & 1 & . & . \\
\hline . & 1 & $\cdot$ & . & . \\
\hline . & . & . & . & 2 \\
\hline 1 & . & . & . & + \\
\hline$\cdot$ & . & $\cdot$ & & . \\
\hline+ & $\cdot$ & $\cdot$ & $\cdot$ & . \\
\hline . & . & $\cdot$ & . & + \\
\hline 1 & $\cdot$ & $\cdot$ & . & . \\
\hline . & . & . & 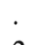 & 1 \\
\hline & 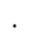 & & 2 & . \\
\hline
\end{tabular}

Diferenciales de subasociación

Ferula tingitana

$\begin{array}{llllll}3 & 3 & 2 & 2 & 3 & 4\end{array}$

\section{Compañeras}

Paronychia argentea

Asphodelus albus

Urginea maritima

Asparagus albus

Malcolmia littorea

Centranthus calcitrapae

Sonchus tenerrimus

Osyris quadripartita

$\begin{array}{llllll}2 & 2 & \cdot & \cdot & 2 & 1 \\ 1 & \cdot & + & + & \cdot & . \\ 1 & \cdot & \cdot & + & \cdot & 1 \\ 1 & 1 & \cdot & + & \cdot & \cdot \\ \cdot & \cdot & + & \cdot & 1 & + \\ \cdot & \cdot & + & \cdot & 4 & 1 \\ 1 & 1 & \cdot & \cdot & \cdot & \cdot \\ \cdot & + & + & \cdot & \cdot & .\end{array}$

Otros táxones: Asteriscus maritimus +, Hirschfeldia incana 1 y Ficus carica + en 2; Silene nicaeensis +, Aetheorhiza bulbosa + , Hypochaeris salzmannii + y Dipcadi serotinum + en 5; Allium ampeloprasum +, Pistacia lentiscus + , Calicotome villosa + , Asparagus aphyllus + y Oxalis pes-caprae 2 en 19; Hedypnois cretica + , Lolium rigidum 2, Rumex bucephalophorus 1, Mercurialis ambigua 1, Silene obtusiflora 1 y Avena barbata 1 en 29; Bromus diandrus 1, Lotus creticus 1 y Euphorbia medicaginea 1 en 31. 
Tabla 5

37: Chenopodietalia-Onopordenea DC, 51-26: Anacyclo radiati-Hordeetum leporini chrysanthemetosum coronarii, 3-39: centaureetosum sonchifoliae Galán de Mera, Cortés \& Sánchez García subass. nova

(Stellarietea, Stellarienea, Sisymbrietalia, Hordeion leporini)

\begin{tabular}{|c|c|c|c|c|c|c|c|c|}
\hline Inventario $n^{\circ}$ & 37 & 3 & 34 & 35 & 36 & 39 & 51 & 50 \\
\hline Area $\mathrm{m}^{2}$ & 50 & 5 & 4 & 4 & 6 & 50 & 20 & 25 \\
\hline Pendiente $(\%)$ & 40 & - & - & - & - & - & - & - \\
\hline
\end{tabular}

Características de asociación y alianza

Hordeum leporinum

Anacyclus radiatus

Hirschfeldia incana

Plantago lagopus

Diferenciales de subasociaciones

Centaurea sonchifolia

Chrysanthemum coronarium

Emex spinosa

Lotus creticus

Características de Sisymbrietalia officinalis

Bromus diandrus

Avena barbata

Lolium rigidum

Bromus madritensis

Medicago polymorpha

Características de Geranio-Cardaminetalia

Rhagadiolus edulis

Geranium purpureum

Geranium rotundifolium

Características de Stellarietea mediae

Erodium malacoides

Galactites tomentosa

Sonchus tenerrimus

Silene colorata

Melilotus segetalis

Lathyrus clymenum

Raphanus raphanistrum

Beta maritima

Chenopodietalia-Onopordenea DC

Urospermum picroides

Achyranthes sicula

Malva sylvestris

Urtica membranacea

Piptatherum miliaceum

Mercurialis ambigua

Glaucium flavum

Calendula suffruticosa

Nicotiana glauca

Sonchus oleraceus

Oxalis pes-caprae

Lolium multiflorum

Solanum nigrum

Antirrhinum cirrhigerum

Euphorbia terracina

Delphinium staphisagria

Echium coincyanum

Compañeras

Daucus maximus

Ferula tingitana

Psoralea bituminosa

Scabiosa atropurpurea

Brachypodium gaditanum

Otros táxones: Dittrichia viscosa + en 3; Dactylis hispanica +, Silene obtusifolia 1 y Lotus arenarius + en 26; Hypochaeris radicata 1, Medicago littoralis 1 y Orobanche ramosa + en 34 ; Ailanthus altissima + en $35 ;$ Galium aparine + en 36; Brachypodium distachyon + en 39; Foeniculum piperitum + y Tropaeolum majus 1 en 50. 
Tabla 6

15-57: Parapholido incurvae-Frankenietum laevis Galán de Mera, Cortés \& Sánchez García ass. nova (Juncetea maritimi, Juncetalia maritimi, Juncion maritimi)

\section{7-28: Polygono-Poetea annuae BC}

$\begin{array}{llllllll}\text { Inventario } \mathrm{n}^{\circ} & 15 & 54 & 55 & 56 & 57 & 27 & 28 \\ \text { Área } \mathrm{m}^{2} & 2 & 4 & 4 & 10 & 20 & 1 & 1\end{array}$

Características de asociación y unidades superiores

Frankenia laevis

Plantago crassifolia

Parapholis incurva

$\begin{array}{lllll}2 & 2 & 2 & 1 & 4 \\ 1 & \cdot & 1 & 1 & 1 \\ 2 & 3 & 4 & 3 & 1\end{array}$

Spergularia marina

\section{Polygono-Poetea annuae $\mathrm{BC}$}

Lolium rigidum

Polycarpon tetraphyllum

Silene colorata

\section{Compañeras}

Crithmum maritimum

Malva sylvestris

Otros táxones: Limonium emarginatum +, Silene obtusifolia + y Desmazeria marina 1, Mesembryanthemum nodiflorum 1 en 15; Scabiosa atropurpurea + en 27; Trifolium gemellum + y Centaurea sonchifolia + en 28; Lagurus ovatus + en 55. 
Tabla 7

16-86: Limonietum emarginati, limonietosum emarginati, helichrysetosum rupestris, limonietosum sinuati Galán de Mera, Cortés \& Sánchez García subass. nova (Crithmo-Staticetea, Crithmo-Staticetalia, Crithmo-Daucion)

13-83: Biscutello sempervirentis-Iberidetum gibraltaricae (Asplenietea, Asplenietalia, Tinguarrenalia, Campanulion velutinae)

\begin{tabular}{lllllllllllllllll}
\hline Inventario no $^{\circ}$ & 16 & 85 & 86 & 40 & 41 & 42 & 52 & 53 & 13 & 77 & 78 & 81 & 82 & 83 & 79 & 84 \\
Área m$^{2}$ & 30 & 10 & 100 & 100 & 100 & 100 & 100 & 100 & 20 & 20 & 20 & 4 & 4 & 4 & 0.5 & 0.5 \\
Orientación & $\mathrm{S}$ & $\mathrm{NE}$ & $\mathrm{S}$ & $\mathrm{NE}$ & $\mathrm{NE}$ & $\mathrm{S}$ & $\mathrm{S}$ & $\mathrm{E}$ & $\mathrm{O}$ & $\mathrm{O}$ & $\mathrm{NE}$ & $\mathrm{NE}$ & $\mathrm{NE}$ & $\mathrm{O}$ & $\mathrm{E}$ & $\mathrm{S}$ \\
Pendiente (\%) & 90 & 30 & 40 & 30 & 40 & 50 & 50 & 50 & 80 & 90 & 90 & 80 & 80 & 90 & 90 & 90
\end{tabular}

Biscutello-Iberidetum gibraltaricae,

Campanulion velutinae y unidades superiores

Iberis gibraltarica

Sedum album

Biscutella sempervirens

Melica minuta

Piptatherum caerulescens

Thymus willdenowii

Cerastium gibraltaricum

Limonietum emarginati, Crithmo-Daucion y

unidades superiores

Asteriscus maritimus

Crithmum maritimum

Silene obtusifolia

Limonium emarginatum

Diferencial de helichrysetosum rupestris

Helichrysum rupestre

Diferencial de limonietosum sinuati

Limonium sinuatum

Aeonium arboreum DC

Aeonium arboreum

Nicotiana glauca

Carpobrotus edulis DC

Carpobrotus edulis

Chrysanthemum coronarium

Pelargonium sp.

Características de Phagnalo-Rumicetea

Echium coincyanum

Rumex induratus

Aloe arborescens DC

Tanacetum cinerariifolium

Aloe arborescens

Agave americana

Asplenietea trichomanis $\mathrm{BC}$

Asplenium trichomanes

Asplenium billotii

Campanula erinus

Anomodonto-Polypodietea $\mathrm{BC}$

Polypodium serrulatum

Senecio cineraria DC

Dactylis hispanica

Suaeda vera

Ecballium elaterium

Beta maritima

Senecio cineraria

Características de Parietarietalia

Umbilicus rupestris

Antirrhinum cirrhigerum

Parietaria diffusa

Compañeras

Sonchus tenerrimus

Calendula suffruticosa

Erodium malacoides

Thapsia villosa

Hyoseris radiata

Lobularia maritima

Ferula tingitana

Daucus maximus

Mercurialis ambigua

Euphorbia squamigera

Lavatera arborea

Scilla peruviana

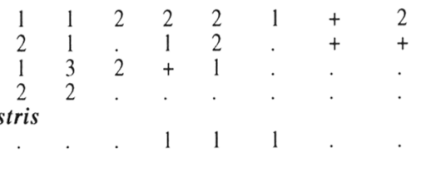

Otros táxones: Psoralea bituminosa + en 13; Prasium majus + en 40; Lolium rigidum + en 42; Malva sylvestris + en 77; Geranium purpureum + en 79; Acanthus platyphyllos + y Vinca difformis + en 83; Galium verrucosum + en 84; Lotus creticus 2, Urospermum picroides 1 y Cichorium endivia + en 86 . 
Tabla 8

Antirrhino cirrhigeri-Parietarietum judaicae Galán de Mera, Cortés \& Sánchez García ass. nova, parietarietosum judaicae, cymbalarietosum muralis Galán de Mera, Cortés \& Sánchez García subass. nova (Asplenietea trichomanis, Parietarietalia, Parietario-Galion)

Inventario $\mathrm{n}^{\circ}$

Area $\mathrm{m}^{2}$

$\begin{array}{lllllllllll}4 & 12 & 44 & 47 & 20 & 21 & 43 & 45 & 46 & 48 & 49 \\ 2 & 20 & 5 & 25 & 20 & 50 & 5 & 50 & 50 & 25 & 25 \\ \mathrm{E} & \mathrm{E} & \mathrm{O} & \mathrm{O} & \mathrm{O} & \mathrm{O} & \mathrm{O} & \mathrm{O} & \mathrm{O} & \mathrm{O} & \mathrm{O}\end{array}$

Orientación

Características de asociación y unidades superiores

Parietaria judaica

Antirrhinum cirrhigerum

Sonchus tenerrimus

Vallantia muralis

Umbilicus rupestris

Phagnalon saxatile

Centranthus ruber

Fumaria capreolata

Ficus carica

Veronica cymbalaria

Sedum album

Melica minuta

Diferenciales de subasociación

Cymbalaria muralis

Trachelium caeruleum

$\begin{array}{lllllllllll}4 & + & 2 & 3 & 1 & 2 & 2 & 1 & + & 2 & 1 \\ 1 & 1 & 1 & 1 & 2 & 1 & + & 2 & 1 & 1 & . \\ . & + & . & . & + & + & . & 1 & . & 1 & 1 \\ . & . & . & 1 & . & . & 1 & 1 & 1 & . & . \\ . & 1 & . & + & . & . & . & . & . & + & . \\ . & 1 & . & . & 1 & . & . & . & . & . & . \\ . & . & . & . & + & 1 & . & . & . & . & . \\ . & . & . & . & . & . & . & 1 & . & 1 & . \\ . & . & . & + & . & . & . & . & . & + & . \\ . & . & . & + & . & . & . & . & . & . & . \\ . & 2 & . & . & . & . & . & . & . & . & . \\ . & + & . & . & . & . & . & . & . & . & .\end{array}$

\section{Características de Geranio-Cardaminetalia}

Fumaria sepium

Geranium purpureum

Centranthus calcitrapae

Características de Chenopodietalia muralis y Stellarietea mediae

Urtica membranacea

Mercurialis ambigua

Erodium malacoides

\section{Tropaeolum majus DC}

Tropaeolum majus

Bromus diandrus

Chrysanthemum coronarium

Medicago polymorpha

Nephrolepis cordifolia DC

Nephrolepis cordifolia
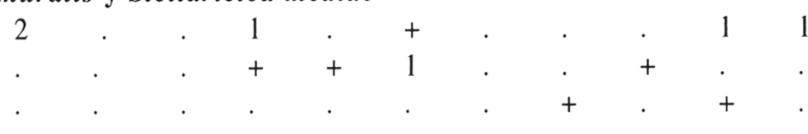

Otros táxones: Daucus maximus + en 4; Prasium majus 1, Oxalis pes-caprae 1, Hyparrhenia hirta + y Galium verrucosum + en 12; Sonchus oleraceus + y Nicotiana glauca + en 20; Ipomoea purpurea 1 y Stellaria pallida + en 21; Piptatherum miliaceum 1 en 45; Scallonia rubra + en 46. 
17. Aloe arborescens DC [Cultivos invasores de aloes y ágaves].

ANOMODONTO-POLYPODIETEA RivasMartínez 1975

\section{Anomodonto-Polypodietea}

[Comunidades empobrecidas con polipodios].

PARIETARIETEA Rivas-Martínez ex Rivas Goday 1964

+ Parietarietalia Rivas-Martínez ex Rivas Goday 1964

* Parietario-Galion murale Rivas-Martínez ex Rivas Goday 1964

19. Antirrhino cirrhigeri-Parietarietum judaicae Galán de Mera, Cortés \& Sánchez García ass. nova [Vegetación fisurícola de muros].

\section{parietarietosum judaicae}

cymbalarietosum muralis Galán de Mera, Cortés \& Sánchez García subass. nova [Vegetación de muros húmedos].

Nephrolepis cordifolia DC [Cultivos invasores de helechos].

Tropaeolum majus DC [Cultivos invasores de capuchina].

\section{BIBLIOGRAFÍA}

ALONSO VARGAS, M.A., M. VICEDO MAESTRE, J. PAYÁ MOYA y A. DE LA TORRE GARCÍA -1998- Sobre el orden Phagnaletalia saxatile Rivas Goday 1964. Itinera Geobot. 11: 159-172.

ASENSI MARFIL, A. -1984- Limonietum emarginati (Crithmo-Limonion) nueva asociación para los sectores Gaditano y Tingitano. Doc. Phytosoc. 8: 45-50.

BARBÉRO, M., P. QUÉZEL \& S. RIVASMARTÍNEZ - 1981- Contribution à l'étude des groupements forestiers et préforestiers du Maroc. Phytocoenologia 9: 311-412.

BARTOLOMÉ, C., M. PEINADO, J.M. MARTÍNEZ PARRAS, F. ALCARAZ, J. ALVAREZ y M. DE LA CRUZ -1989- Esquema sintaxonómico de la vegetación nitrófila de Andalucía (España). IX Jornadas de Fitosociología. Alcalá de Henares.

BENABID, A. -1984- Étude phytoecologique des peuplements forestiers et préforestiers du Rif centro-occidental (Maroc). Trav. Inst. Sci. Chérifien, Sér. Bot. 34: 1-64.

BIONDI, E. -1989- Smyrnium olusatrum L. vegetation in Italy. Braun-Blanquetia 3: 219222.

BOLÒS, O. -1957- De vegetatione valentina, II. Anales Inst. Bot. Cavanilles 32(2): 477-488.

BOLÒS, O. -1962- El paisaje vegetal barcelonés. Facultad de Filosofía y Letras. Barcelona.

BOLÒS, O. \& R. MOLINIER -1958-Recherches phytosociologiques dans l'Ile de Majorque. Collect. Bot., Barcelona 5: 699-865.

BRAUN-BLANQUET, J. -1964Pflanzensoziologie. Grundzüge der Vegetationskunde. Springer. Wien.

BRULLO, S. \& C. MARCENÒ -1985- Contributo alla conoscenza della vegetazione nitrofila della Sicilia. Coll. Phytosoc. 12: 23-148.

CANTÓ, P., S. LAORGA y D. BELMONTE -1986Vegetación y catálogo florístico del Peñón de Ifach (Penyal d'Ifac)(Alicante, España). Opusc. Bot. Pharm. Complutensis 3: 3-86.

CASTROVIEJO, S. (coord.) -1986-1999- Flora iberica. CSIC. Madrid.

CORTÉS, J.E. -1996-The history of the vegetation of Gibraltar. Almoraima 15: 39-50.

CORTÉS, J.E. \& C. FINLAYSON -1988- The flowers and wildlife of Gibraltar. Gibraltar Books Ltd. Gibraltar.

CORTÉS, J.E. \& L. LINARES - 1993- The Gibraltar campion Silene tomentosa Otth in DC.: probable extinction of a Gibraltar endemic. Alectoris 8: 64-65.

DAUMAS, P., P. QUÉZEL \& S. SANTA -1952-: Contribution à l'étude des groupements végétaux rupicoles de l'Oranie. Bull. Soc. Hist. Nat. Afr. Nord 43: 186-202.

DEBEAUX, M. \& M.G. DAUTEZ - 1889- Synopsis de la Flore de Gibraltar. Paris-Gibraltar.

DEIL, U. -1994- Felsgesellschaften beiderseits der Straße von Gibraltar. Hoppea, Denkschr. Regensb. Bot. Gess. 55: 757-814.

DEIL, U. -1997-Zur geobotanischen Kennzeichnung von Kulturlandschaften. Franz Steiner. Stuttgart. DEIL, U. \& M. HAMMOUMI - 1997- Contribution 
à l'étude des groupements rupicoles des Bokkoya (Littoral du Rif Central, Maroc). Acta Bot. Malacitana 22: 131-146.

DIDON, J., M. DURAND-DELGA \& J. KORNPROBST -1973- Homologies gèologiques entre les deux rives du détroit de Gibraltar. B.S.G.F. 15 (7): 76-104.

DIERSCHKE, H. -1993-Grundlagen und Methoden der Pflanzensoziologie. Ulmer. Stuttgart.

FERNÁNDEZ CASAS, J. -1972- Notas fitosociológicas breves, II. Trab. Dep. Bot. Univ. Granada 1: 21-57.

FERRO, G. - 1980- La vegetazione di Butera (Sicilia meridionale). Atti Ist. Bot. Lab. Crittog. Univ. Pavia 13: 51-116.

FOUCAULT, B. -1981-Réflexions sur l'appauvrissement des syntaxons aux limites chorologiques des unités phytosociologiques supérieurs et quelques-unes de leurs consequences. Lazaroa 3: 75-100.

GALÁN DE MERA, A. -1993-Flora y vegetación de los términos municipales de Alcalá de los Gazules y Medina Sidonia (Cádiz, España). Tesis Doctoral. Universidad Complutense. Madrid.

GALÁN DE MERA, A. -1995- Las formaciones de Calicotome villosa (Poiret) Link en el SW de la Península Ibérica y $\mathrm{N}$ de África. Bot. Complut. 20: 81-87.

GALÁN DE MERA, A., E. DE CASTRO \& J.A. VICENTE ORELLANA - 1999 a- Hypochaeris alliatae group (Asteraceae) in the western Mediterranean Region. Nordic J. Bot. 19: 587595.

GALÁN DE MERA, A., J.E. CORTÉS, J.A. VICENTE ORELLANA y R. MORALES ALONSO -1999 b- Silene gazulensis sp. nov. (Caryophyllaceae): un nuevo endemismo del entorno del Estrecho de Gibraltar. Acta Bot. Malacitana 24: 237-262.

GALÁN DE MERA, A., M.A. HAGEN y J.A. VICENTE ORELLANA - 1996- Variabilidad de las comunidades rupícolas brio-pteridofíticas en el SO de la Península Ibérica y NO de África. Orsis 11: 7-13.

GALÁN DE MERA, A., I. SÁNCHEZ GARCÍA \& J.A. VICENTE ORELLANA -1997- Coastal plant communities of the southwestern Iberian Peninsula, Spain and Portugal. Phytocoenologia 27: 313-352.
GARCÍA RÍO, R. y F. NAVARRO ANDRÉS - 1994 Flora y vegetación cormofíticas de las comarcas zamoranas del Pan, Tera y Carballeda. Stud. Bot. Univ. Salamanca 12: 23-202.

GÉHU, J.M. \& N. SADKI -1995- Remarques de phytosociologie et de synchorologie comparées sur le littoral Algerois. Doc. Phytosoc. 15: 341 357.

GREUTER, W., H.M. BURDET \& G. LONG -1989Med-Checklist 4. Dicotyledones (LauraceaeRhamnaceae). Genève.

JAHANDIEZ, E. \& R. MAIRE -1931-1934Catalogue des plantes de Maroc. Minerva. Alger.

JEANMONOD, D. -1984- Révision de la section Siphonomorpha Otth du genre Silene L. (Caryophyllaceae) en Méditerranée occidentale III: aggrégat italica et espèces affines. Candollea 39: 549-639.

KELAART, E.F. -1846- Botany and topography of Gibraltar. London.

KIEFER, H. \& G. BOCQUET -1979- Silene velutina Pourret ex Loiseleur (Caryophyllaceae)example of a messinian destiny. Candollea 34 : 459-472.

KITAYAMA, K. \& D. MUELLER-DOMBOIS 1995- Biological invasion on an oceanic island mountain: Do alien plant species have wider ecological ranges than native species? J. Veg. Sci. 6: 667-674.

KOPECKÝ, K., J. DOSTALEK \& T. FRANTIK 1995- The use of the deductive method of syntaxonomic classification in the system of vegetational units of the Braun-Blanquet approach. Vegetatio 117: 95-112.

LINARES, L., A. HARPER \& J. CORTÉS -1996The Flowers of Gibraltar. Flora Calpensis. Rueda. Madrid.

LOIDI, J. y A. GALÁN DE MERA - 1988- Datos sobre la vegetación rupícola de la comarca madrileña de Torrelaguna. Stud. Bot. Univ. Salamanca 7: 159-171.

MARTÍNEZ PARRAS, J.M. -1982- Parietario mauritanicae-Ceratocapnetum heterocarpae ass. nova. Anales Jard. Bot. Madrid 39: 187-190.

MATUSKIEWICZ, W. \& A. -1981- Das Prinzip der Mehrdimensionalen gliederung der Vegetationseinheiten, erläutet am Beispiel der Einchen-Hainbuchenwälder in Polen. In Dierschke, H. (ed.): Syntaxonomie.- Ver. Int. 
Symp. Veget. K. de Rintelen, 123-145.

MELÉNDEZ, B. y J.M. FUSTER -1984-Geología. $4^{\text {a }}$ ed. Paraninfo. Madrid.

NÈGRE, R. -1957- Les Frankenia du Maroc. Trav. Inst. Sci. Chérifien, Sér. Bot. 12: 7-33.

NÈGRE, R. -1964- Carte au 1/50.000 de Tipasa. Notes Doc. Inst. Cartogr. Vég. Algérie 1: 1-68.

ORTIZ, S. \& J. RODRÍGUEZ-OUBIÑA -1993Synopsis of the Rupicolous Vegetation of Galicia (North-western Iberian Peninsula). Folia Geobot. Phytotax. 28: 15-49.

PÉREZ LATORRE, A.V., A. GALÁN DE MERA, U. DEIL y B. CABEZUDO - 1996- Fitogeografía y vegetación del sector Aljíbico (Cádiz-Málaga, España). Acta Bot. Malacitana 21: 241-267.

PÉREZ LATORRE, A.V., A. GALÁN DE MERA, P. NAVAS, D. NAVAS, Y. GIL y B. CABEZUDO -1999- Datos sobre la flora y vegetación del Parque Natural de Los Alcornocales (Cádiz-Málaga, España). Acta Bot. Malacitana 24: 133-184.

PÉREZ LATORRE, A.V., J.M. NIETO CALDERA y B. CABEZUDO -1993- Contribución al conocimiento de la vegetación de Andalucía II.- Los alcornocales. Acta Bot. Malacitana 18: 223-258.

RIVAS GODAY, S. -1964-Vegetación y flórula de la cuenca extremeña del Guadiana. Diputación Provincial de Badajoz. Madrid.

RIVAS GODAY, S. y A. RIGUAL -1958- Algunas asociaciones de la provincia de Alicante. Anales Inst. Bot. Cavanilles 16: 538-548.

RIVAS GODAY, S. y S. RIVAS-MARTÍNEZ 1967- Matorrales y tomillares de la Península Ibérica comprendidos en la clase OnonidoRosmarinetea. Anales Inst. Bot. Cavanilles 25: 5-201

RIVAS-MARTÍNEZ, S. -1969- Vegetatio Hispaniae. Notula I. Publ. Inst. Biol. Aplicada 46: 5-34.

RIVAS-MARTÍNEZ, S. -1974- La vegetación de la clase Quercetea ilicis en España y Portugal. Anales Inst. Bot. Cavanilles 31: 205-259.

RIVAS-MARTÍNEZ, S. -1978- La vegetación del Hordeion leporini en España. Doc. Phytosoc. 2: 377-391.

RIVAS-MARTÍNEZ, S., J.C. BÁSCONES, T.E. DÍAZ, F. FERNÁNDEZ GONZÁLEZ y J. LOIDI -1991- Vegetación del Pirineo occidental y Navarra. Itinera Geobot. 5: 5-456.
RIVAS-MARTÍNEZ, S., M. COSTA, S. CASTROVIEJO y E. VALDÉS -1980Vegetación de Doñana (Huelva, España). Lazaroa 4: 5-189.

RIVAS-MARTÍNEZ, S., M. LOUSA, T.E. DÍAZ, F. FERNÁNDEZ-GONZÁLEZ y J.C. COSTA 1990- La vegetación del sur de Portugal (Sado, Alentejo y Algarve). Itinera Geobot. 3: 5-126. ROSE, E.P.F. \& M.S. ROSENBAUM -1990- Royal engineer geologists and the geology of Gibraltar. Gibraltar.

VALDÉS, B., S. TALAVERA y E.F. GALIANO (eds.) -1987- Flora Vascular de Andalucía Occidental. Ketres. Barcelona.

Aceptado para su publicación en mayo de 2000

Dirección de los autores. A. GALÁN DE MERA: Laboratorio de Botánica, Universidad San PabloCEU, Apartado 67, 28660- Boadilla del Monte (Madrid, España). J. E. CORTÉS: Gibraltar Botanic Gardens, The Alameda, Red Sands Road, P.O. Box 843, Gibraltar (Gran Bretaña). Í. SÁNCHEZ GARCÍA: Parque Zoológico y Jardín Botánico «Alberto Durán», Taxdirt s/n, 11404- Jerez de la Frontera (Cádiz, España). 